\title{
A Dynamical Analysis on a Tumour Virotherapy Model with Standard Incident Rate
}

\author{
Deasy Sandhya Elya Ikawati*, Wuryansari Muharini Kusumawinahyu, Trisilowati
}

Department of Mathematics, Brawijaya University, Malang, Indonesia

\section{ABSTRACT}

This paper discusses a dynamical analysis on a model that governs the growth of tumour cell under a therapy by using oncolytic viruses, on the standard incident rate. The model is a modification of the similar one by replacing the bilinear incident rate with the standard one. The conducted dynamical analysis consists of the determination of equilibrium points and their existence conditions, followed by local as well as global stability analysis of the equilibrium points. The analytical result shows that there are two equilibrium points, namely uninfected and the endemic point, which needs a condition to exist. Stability analysis shows that there is a dimensionless basic reproduction number that marks the existence as well as the stability of equilibrium points. When basic reproduction number is less than one, there is only the uninfected equilibrium, which is global asymptotically stable. On the other hands, both of equilibrium points exist when the basic reproduction number is more than one, but the uninfected point is not stable anymore, while the endemic one is local asymptotically stable under a condition. Some numerical simulations are performed to illustrate the analytical result. Numerically, it can also be demonstrated that there is a set of parameters which indicates that tumour can be fully removed.

Keywords: Tumour, oncolytic virotherapy, standard incident rate, stability analysis

\section{INTRODUCTION}

The tumour is a disease in the form of abnormal lumps or swellings. Most of the tumour is caused by neoplasm, a condition caused by the rapid division of cells that have undergone some form of mutation. One of the tumour treatments is applying therapy by using the oncolytic virus. The virus can infect the tumour cell genetically and replicates itself without damage the normal cell [1].

In 2003, Wodarz proposed a model of tumour growth under oncolytic virus therapy in the form of three-dimensional differential equation [2]. The three dependent variables in the model respectively represent the population density of uninfected tumour cells, infected tumour cells, and independent virus in the human body. Dingli et al. modified the model proposed by Wodarz by adding the assumption that the death rate of uninfected tumour cells is contained in the logistic growth rate [3]. Furthermore, the natural death rate and the other death rate of tumour cells caused by

\section{*Corresponding author:}

Wuryansari Muharini Kusumawinahyu

Department of Mathematics, Faculty of Mathematics and Natural

Sciences, Brawijaya University

Jalan Veteran, Malang, Indonesia 65145

E-mail: wmuharini@ub.ac.id virus infection are considered as a unity called infected tumour cells death rate. In 2008, Dingli et al. modified their previous model by adding two assumptions, namely the process of fusion between tumour cells and the oncolytic virus is resulting infected tumour cells and each virus is assumed to be dead after infecting tumour cells [4]. Moreover, Tian modified the model proposed by Dingli, et al., by adding a parameter that shows the burst size of virus $[1,4]$.

It has to be noted that all of above models applied bilinear incident rate as the rate of oncolytic virus infection. Nevertheless, the research carried out by Min et al. (2008) about the general model for virus infection concludes that bilinear infection rate is not realistic since the incident rate is indicated as the source of the burst size of the virus, which can exceed the available cells. Consequently, Min et al. suggested using standard incident rate instead of the bilinear one [5].

Hence, in this paper, we investigated the dynamics of the modified model of Tian by changing the bilinear

\section{How to cite:}

Ikawati DSE, Kusumawinahyu WM, Trisilowati (2016) A

Dynamical Analysis on a Tumour Virotherapy Model with Standard Incident Rate. J. Trop. Life. Science 7 (1): 16 - 22. 
infection incident rate into standard infection incident rate. Dynamical analysis is employed by investigating the existence condition and the stability of equilibrium points. The results from some numerical simulations are presented to illustrate the analysis result [1].

\section{MATERIALS AND METHODS}

This work is performed by conducting several steps, namely modification of the mathematical model, determination of equilibrium points and their existence conditions, observation on the local as well as global stability of the equilibrium points, and numerical simulations supporting the analytical result.

\section{Mathematical model}

We refer to a model of oncolytic virus infection on tumour investigated in Tian [1].

$$
\begin{aligned}
& \frac{d x}{d t}=r x\left(1-\frac{x+y}{K}\right)-\beta x v \\
& \frac{d y}{d t}=\beta x v-\delta y \\
& \frac{d v}{d t}=b \delta y-\gamma-\beta x v
\end{aligned}
$$

The $\chi, y$, and $v$ represent the number of uninfected tumour cells, the number of infected tumour cells by the oncolytic virus, and the number of the free oncolytic virus in the human body, respectively. The uninfected tumour cells grow logistically under growth rate $r$ and carrying capacity $\mathrm{K}$. The tumour cells are infected by oncolytic virus under bilinear incident rate $\beta \chi y$ so that the uninfected tumour cells become infected cells. Hence, the uninfected tumour cells are reduced, while the number of infected tumour cells is increased. The number of virus is also decreased since Tian assumed that the virus dies after infecting tumour cells. The natural death rate of infected tumour cells is governed by parameter $\delta$, while the number of virus increased proportionally with the death rate of infected tumour cell under burst parameter $b$. Here, $\gamma$ represents the natural death rate of the virus.

\section{Determination of the equilibrium points}

Consider an autonomous dynamical system,

$$
\begin{aligned}
& \frac{d x}{d t}=f(x, y, z) \\
& \frac{d y}{d t}=g(x, y, z) \\
& \frac{d z}{d t}=h(x, y, z)
\end{aligned}
$$

the equilibrium point of (2), denoted by $E=\left(\chi^{*}, y^{*}, v^{*}\right)$, is the solution of equation (2) satisfying $\frac{d x}{d t}=\frac{d y}{d t}=\frac{d z}{d t}=0$ [6]. When the system (2) represents the growth of populations, $\chi^{*}, \mathrm{y}^{*}$, and $v^{*}$ need to be non-negatives. This condition will determine the existence condition for the equilibrium point.

\section{Observing the stability of the equilibrium point}

When the system (2) is a nonlinear system, it is not easy to obtain its exact solution. One way to understand the behaviour of the solutions of system (2) is by investigating the local as well as the global stability of equilibrium points. Local stability analysis can be performed by linearizing system (2) around its equilibrium point, resulting to an approximating linear system

$$
\frac{d}{d t} \vec{w}=J \vec{w}
$$

where $\vec{w}=(x, y, z)-\left(x^{*}, y^{*}, z^{*}\right)$ and $\mathrm{J}$ is Jacobian matrix at $E=\left(\chi^{*}, y^{*}, v^{*}\right)$, namely

$$
J=\left(\begin{array}{lll}
\frac{\partial f}{\partial x}(E) & \frac{\partial f}{\partial y}(E) & \frac{\partial f}{\partial y}(E) \\
\frac{\partial g}{\partial x}(E) & \frac{\partial g}{\partial x}(E) & \frac{\partial g}{\partial x}(E) \\
\frac{\partial h}{\partial x}(E) & \frac{\partial h}{\partial x}(E) & \frac{\partial h}{\partial x}(E)
\end{array}\right)
$$

The stability of $E=\left(\chi^{*}, y^{*}, v^{*}\right)$ is determined by the sign of the real parts of the eigen values of matrix J. $E=$ $\left(\chi^{*}, y^{*}, v^{*}\right)$ is local asymptotically stable when all of the real parts of the eigen values of matrix $J$ are negatives [7].

One way to perform global stability analysis on $E=$ $\left(\chi^{*}, y^{*}, v^{*}\right)$ is by defining a scalar Lyapunov function for system (2). Let $\Omega \subseteq \mathbf{R}^{3}$ is a neighbourhood of $E=$ $\left(\chi^{*}, y^{*}, v^{*}\right) . \quad L(\chi, y, v)$ is called as a weakly Lyapunov function for $E=\left(\chi^{*}, y^{*}, v^{*}\right)$ when it satisfies

$$
\begin{aligned}
& \text { (a) } L(E)=0 \text { and } L(\vec{x})>0 \forall \vec{x} \neq \mathrm{E} \text { in } \Omega \text {, } \\
& \text { (b) } L^{\prime}(\vec{x}) \leq 0 \forall \vec{x} \in \Omega \text {. }
\end{aligned}
$$

Furthermore, when the condition (b) is replaced by $L^{\prime}(\vec{x})<0 \forall \vec{x} \neq$ Ein $\boldsymbol{\Omega}$ then $\mathrm{L}$ is called strong Lyapunov function. The equilibrium point $\mathrm{E}$ is globally stable when there is a weakly Lyapunov function for E. Otherwise; the equilibrium point $\mathrm{E}$ is global asymptotically stable when there is a strong Lyapunov function for $\mathrm{E}$ [8]. 


\section{Numerical simulations}

Some numerical simulations are conducted by using the Fourth Order - Runge Kutta Method apllied in Matlab software. To illustrate the analytical result, some scenarios of simulations are presented by choosing some sets of parameters values.

\section{RESULTS AND DISCUSSION}

\section{Mathematical model}

In this paper, we modify the model of Tian [1] by changing the bilinear incident rate into standard incident rate as suggested by Min et al. [5]. This modification is based on the fact that the infection rate depends on the availability of the cells number. The more realistic modified model investigated in this paper is as follows.

$$
\begin{aligned}
& \frac{d x}{d t}=r x\left(1-\frac{x+y}{K}\right)-\frac{\beta x v}{x+y} \\
& \frac{d y}{d t}=\frac{\beta x v}{x+y}-\delta y \\
& \frac{d v}{d t}=b \delta y-\gamma v-\frac{\beta x v}{x+y^{\prime}}
\end{aligned}
$$

\section{Determination and existence of the equilibrium point}

The equilibrium point of (6) is reached when the system (6) in the steady state condition, namely when the system does not change in time anymore [6]. It means that the differential is zero. Hence, we obtain the point by solving the following system of equation

$$
\begin{aligned}
& r x\left(1-\frac{x+y}{K}\right)-\frac{\beta x v}{x+y}=0 \\
& \frac{\beta x v}{x+y}-\delta y=0, \\
& b \delta y-\gamma-\frac{\beta x v}{x+y}=0
\end{aligned}
$$

We found that there are two equilibrium points, namely $E_{1}=(K, 0,0)$ and $E_{2}=\left(\chi^{*}, y^{*}, v^{*}\right)$, where

$$
\begin{aligned}
x^{*} & =\frac{K(r \gamma-\delta \beta b+\delta \beta+\delta \gamma)}{r \beta(b-1)} \\
y^{*} & =\frac{\chi^{*}(\beta(b-1)-\gamma)}{\gamma} \\
v^{*} & =\frac{K \delta(r \gamma-\delta \beta b+\delta \beta+\delta \gamma)(\beta b-\gamma-\beta)}{r \beta \gamma^{2}}
\end{aligned}
$$

The complicated form of equilibrium point $E_{2}=\left(\chi^{*}, y^{*}\right.$, $\left.v^{*}\right)$ needs the investigation on its existence. The result of the investigation is briefly stated in the following proposition.
Proposition 1: The equilibrium point $E_{2}$ exists when b satisfies the following condition

$$
1+\frac{\gamma}{\beta}<b<1+\frac{\gamma}{\beta}\left(1+\frac{r}{\delta}\right) .
$$

\section{The stability of the equilibrium point}

The investigation of the local stability of equilibrium points of a nonlinear system is performed by linearizing the system around the point. This process is resulting a Jacobian matrix of the system, which can be used to determine the stability by considering the eigen values of the matrix. When all of the eigen values are negative then the equilibrium point is stable. Otherwise, it is unstable. The Jacobian matrix of the system (6) is

$J=\left(\begin{array}{ccc}r-\frac{2 r x+r y}{K}-\frac{\beta v}{x+y}+\frac{\beta x}{(x+y)^{2}} & -\frac{r x}{K}+\frac{\beta x v}{(x+y)^{2}} & \frac{-\beta x}{x+y} \\ \frac{\beta y v}{(x+y)^{2}} & -\frac{\beta x v}{(x+y)^{2}}-\delta & \frac{\beta x}{x+y} \\ -\frac{\beta y v}{(x+y)^{2}} & \delta b+\frac{\beta x v}{(x+y)^{2}} & -\gamma-\frac{\beta x}{x+y}\end{array}\right)$,

The Stability of $E_{1}=(K, 0,0)$

Jacobian matrix at $E_{1}=(K, O, O)$ is

$$
J\left(E_{1}\right)=\left(\begin{array}{ccc}
-r & -r & -\beta \\
0 & -\delta & \beta \\
0 & \delta b & -\gamma-\beta
\end{array}\right) .
$$

The stability of the equilibrium point $E_{1}$ is determined by the sign of the real part of the eigen values of $J\left(E_{1}\right)$. If they are negative, then $E_{1}$ is local asymptotically stable. It is clear that one of the eigenvalues is $\lambda_{1}=-r$, while the other two eigenvalues belongs to matrix $A=\left[\begin{array}{cc}-\delta & \beta \\ \delta b & -\gamma-\beta\end{array}\right]$. By investigating the sign of $\operatorname{det}(A)$ and $\operatorname{trace}(A)$ [9], it is not difficult to show that the equilibrium point $E_{1}$ is local asymptotically stable when $\frac{\beta b-\gamma}{\beta}<1$ About the existence of $E_{2}$ stated in Proposition 1, we can conclude that $E_{2}$ does not exist when $E_{1}$ is local asymptotically stable.

In the epidemic model, there is a number so-called Basic Reproduction Number, denoted by $R_{0}$, which indicates the occurrence of infection leads to an epidemic situation [10]. This number represents how many tumour cells are infected by one previous infected tumour cell. The value of $R_{0}$ more than one indicates that more than one tumour cells are infected by one old infected cell. It means that the number of tumour cells infected by the old cells is increasing. The condition when equilibrium $E_{1}$ is stable describes the situation when the virus fails to infect the tumour cells, since at 
$E_{1}$ we have $\chi=0, y=0$, and $v=0$. Since this condition happens when $\frac{\beta b-\gamma}{\beta}<1$, it can be concluded that the basic reproduction number for our model is $R_{0}=\frac{\beta b-\gamma^{-}}{\beta}$. It is readily seen that this number is dimensionless. Un the other hand, when $R_{0}=\frac{\beta b-\gamma}{\beta}>1$, which represents the existence of equilibrium point $E_{2}$, we can say that the tumour cells are infected by the oncolytic virus such that the tumour cells are reduced. So, in the medical point of view, the condition when $R_{o}>1$ is the expected situation.

Compared to Tian (2011) model, the basic reproduction number $R_{0}$ of our model is dimensionless and does not depend on the maximum limit of tumour cells number $\mathrm{K}$ anymore [1]. This is the important result comes from the modification of bilinear into standard incident rate infection, which is the proportion of the tumour cell number.

Furthermore, the global stability analysis of equilibrium $E_{1}=(K, O, O)$ is also performed by defining Lyapunov function

$$
L=\frac{1}{2} \beta b(\beta+\gamma) y^{2}+\beta^{2} b y v+\frac{1}{2} \beta^{2} v^{2} .
$$

It can be verified that the Lyapunov function (12) satisfies the following conditions when $R_{o}<1$.

a. $L(K, 0,0)=0$,

b. $L(x, y, v)>0$ for each $(x, y, v) \neq(K, 0,0)$,

c. $\frac{d L}{d t}(x, y, v)<0$ for each $(x, y, v) \neq(K, 0,0)$.

Hence, it can be concluded that $E_{1}$ is asymptotically global stable when $R_{0}>1$.

The Stability of $E_{2}=\left(\chi^{*}, y^{*}, v^{*}\right)$

The Jacobian matrix at $E_{2}=\left(\chi^{*}, y^{*}, v^{*}\right)$ is

$$
J\left(E_{2}\right)=\left(\begin{array}{lll}
A & B & C \\
D & E & F \\
G & H & I
\end{array}\right),
$$

where

$$
\begin{aligned}
& A=B=\frac{2 \gamma \beta b-2 \gamma \delta-r \gamma-2 \delta \beta}{\beta(b-1)}, C=-\frac{\gamma}{b-1}, \\
& D=\frac{\delta(-\beta b+\beta+\gamma)^{2}}{\gamma \beta(b-1)}, E=\frac{\delta(-2 \beta b+2 \beta+\gamma)}{\beta(b-1)}, \\
& F=\frac{\gamma}{b-1}, G=-D, \\
& H=\frac{\delta\left(\beta b^{2}-\gamma-\beta\right)}{\beta(b-1)}, \text { and } I=-\frac{b \gamma}{b-1} .
\end{aligned}
$$

The characteristic polynomial for $\mathrm{J}\left(E_{2}\right)$ is

where

$$
\lambda^{3}+A_{1} \lambda^{2}+A_{2} \lambda+A_{3}=0
$$

$$
\begin{gathered}
A_{1}=-A-E-I \\
A_{2}=E A+I A+E I-H F-B D-C G \\
A_{3}=-E I A+A H F+B D I-B F G-C D H+C E G .
\end{gathered}
$$

According to Routh-Hurwitz criteria in Murray (2002), the characteristic polynomial (16) has negative roots if only if $A_{1}>O, A_{3}>O$ and $A_{1} A_{2}-A_{3}>O$ [11]. Hence, the local stability of $E_{2}=\left(\chi^{*}, y^{*}, v^{*}\right)$ is stated in the following proposition.

Proposition 2: If $\mathrm{R}_{0}>1$, equilibrium point $E_{2}=\left(\chi^{*}, y^{*}\right.$, $\left.v^{*}\right)$ is local asymptotically stable when $A_{1} A_{2}-A_{3}>0$.

\section{Numerical simulations}

Three numerical simulations are executed by constructing a scheme in Matlab software to illustrate the analytical result. The first simulation is aimed to show the stability of equilibrium $E_{1}=(K, O, O)$ when $\mathrm{R}_{0}>1$, while the second and the third simulations show the conditions when $E_{2}=\left(\chi^{*}, y^{*}, v^{*}\right)$ exist. The second simulation shows the situation when $E_{2}=\left(\chi^{*}, y^{*}, v^{*}\right)$ is stable, while the third simulation shows when both of $E_{1}$ and $E_{2}$ are unstable.

In order to show the global stability of equilibrium point $E_{1}$, a simulation is made using a set of parameters, namely $\mathrm{r}=0.2, \mathrm{~K}=2139, \beta=0.0127, \delta=0.01, \gamma=$ 0.125 , and $\mathrm{b}=10$, to obtain $\mathrm{R}_{0}=0.157<1$. There is only one equilibrium point $E_{1}$, since the existence condition of $E_{2}$ is not fulfilled, namely $1+\frac{\gamma}{\beta}=10.84>10$ $=\mathrm{b}$. The numerical simulation uses six initial values, namely $\mathrm{N}_{1}=(200,1000,800), \mathrm{N}_{2}=(100,7000,800), \mathrm{N}_{3}$ $=(1000,500,50), \mathrm{N}_{4}=(500,0,0), \mathrm{N}_{5}=(1000,0,0)$, and $\mathrm{N}_{6}=(1800,0,0)$.

The result of numerical simulation provided in Figure 1 shows that all the orbits that start from the six initial conditions converge to the equilibrium point $E_{1}$ $=(2139,0,0)$. This result is in accordance with the analytical result saying that $E_{1}$ is global asymptotically stable.

The curves of solutions that show the number of uninfected tumour inside the human body every time until $t=900$ is presented in Figure $1 b$. The curves are indicated by their initial values of the total tumour cells, namely the sum of the first and second component of $\mathrm{N}_{1}, \mathrm{~N}_{2}, \mathrm{~N}_{3}, \mathrm{~N}_{4}, \mathrm{~N}_{5}$, and $\mathrm{N}_{6}$ coordinates. For example, the blue curve describes how the number of the total tumour cells which start from the initial number of the total tumour cell $\mathrm{N}_{1}$, namely $200+1000=1200$, increases to the equilibrium of the total tumour cell $\mathrm{E}_{1}$, namely 2139 . 


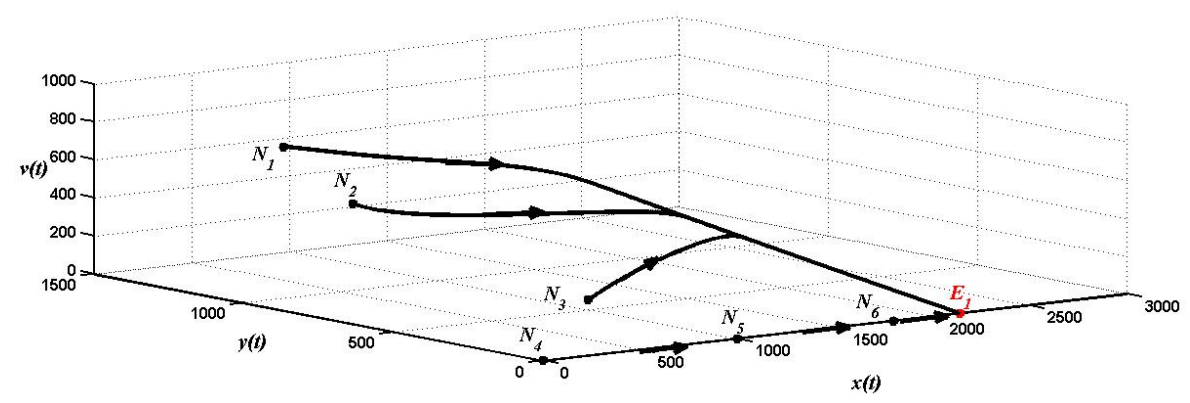

(a)

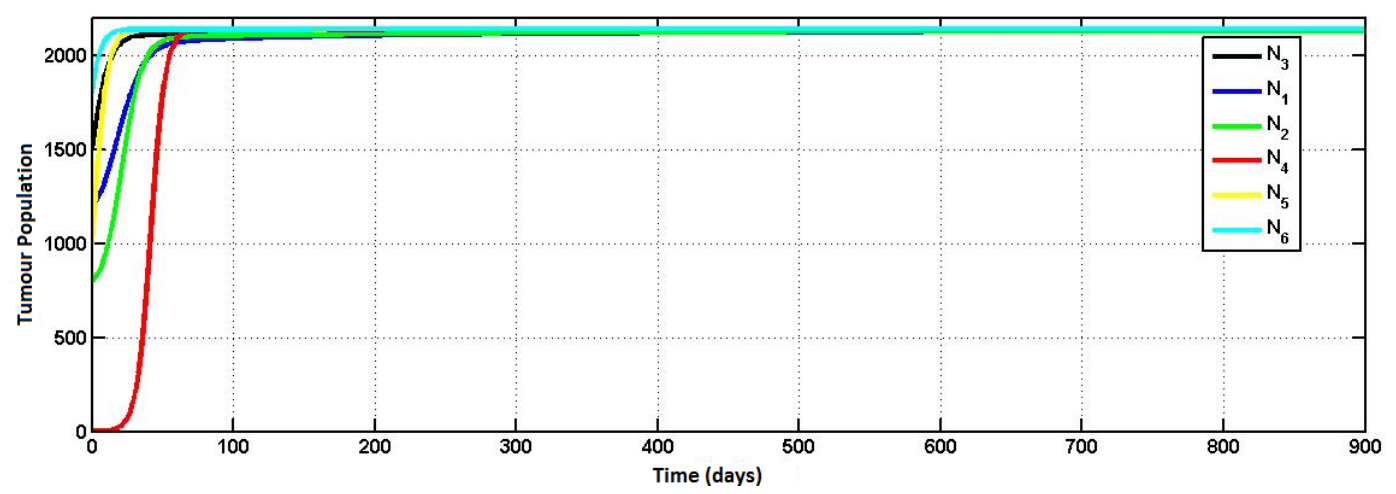

(b)

Figure 1. The phase portrait of system (6) shows that E1 is asymptotically stable (a). Number of total tumour cells every time, for 0 $\leq \mathrm{t} \leq 900(\mathrm{~b})$

It can be seen that along the interval time $[0,900]$ the number of total tumour cells, including uninfected and infected tumour cell, never exceeded the maximum limit of tumour cell inside the human body, $\mathrm{K}=2139$. It is in accordance with the motivation of the implementation of standard incident rate to the model. The stability of equilibrium point $E_{1}$ implies that the population of infected tumour cell and free virus vanish. The condition can be interpreted as a condition where the virus cannot infect the tumour cell. Therefore, from the medical point of view, the therapy using the oncolytic virus failed.

The second simulation is executed to show a situation when the equilibrium point $E_{2}$ is local asymptotically stable. In order to satisfy the existence and the stability conditions of $E_{2}$, we pick a set of parameter values, namely $r=0.2, K=2139, \beta=0.0127, \delta=0.01$, $\gamma=0.125$ and $b=20$. Hence, there are two equilibrium, namely $E_{1}=(2139,0,0)$ and $E_{2}=(1056,982,1494)$. It can be verified that $R_{0}=10.16>1$ and $A_{1} A_{2}-A_{3}=$ $0.00343>0$.

The initial values used in this simulation are $N_{1}=$ (200, 1000, 800), $N_{2}=(100,700,600), N_{3}=(650,500$, $0), N_{4}=(1000,500,50), N_{5}=(500,0,0), N_{6}=(1000,0$, $0)$, and $N_{7}=(1800,0,0)$.
The result of the simulation is presented in Figure 2a. It can be seen that four orbits start from $N i ; i=1$, 2, 3, 4 tend to reach the equilibrium point $E_{2}=(1056$, $982,1494)$. Nevertheless, three orbit start from some position at the x-axis, namely $N i ; i=5,6,7$, go to equilibrium point $E_{1}$, instead of going to $E_{2}$, even though the parameter values do not satisfy the stability condition of equilibrium $E_{1}$. It looks like a contradiction, but if we see more detail, it is easy to see that the eigen values of Jacobian matrix at equilibrium point $E_{1}$ are $\lambda_{1}=-$ $0.2, \lambda_{2}=0.0075$, and $\lambda_{3}=-0.155$, with the corresponding eigenvectors are $\xi_{1}=(1,0,0), \xi_{2}=(-0.421,0.403$, $0.915)$, and $\xi_{3}=(0.462,-0.432,1.499)$ respectively. Hence, the space spanned by $\xi_{1}=(1,0,0)$, which is $\mathrm{x}$ axis, is a stable manifold. Therefore, every orbit that starts from any position at positive $\mathrm{x}$-axis, namely $(\chi, O$, $O), O<\chi<K$, will tend to $E_{1}$. It supports the analytical result, which says that $E_{2}$ is not global asymptotically stable, but only local asymptotically stable. From the medical point of view, we can propose a set of parameter values which can be used to reduce the number of tumour cell. But if Virotherapy is not applied then the tumour cell will grow to the maximum capacity of tumour cell in the human body. 


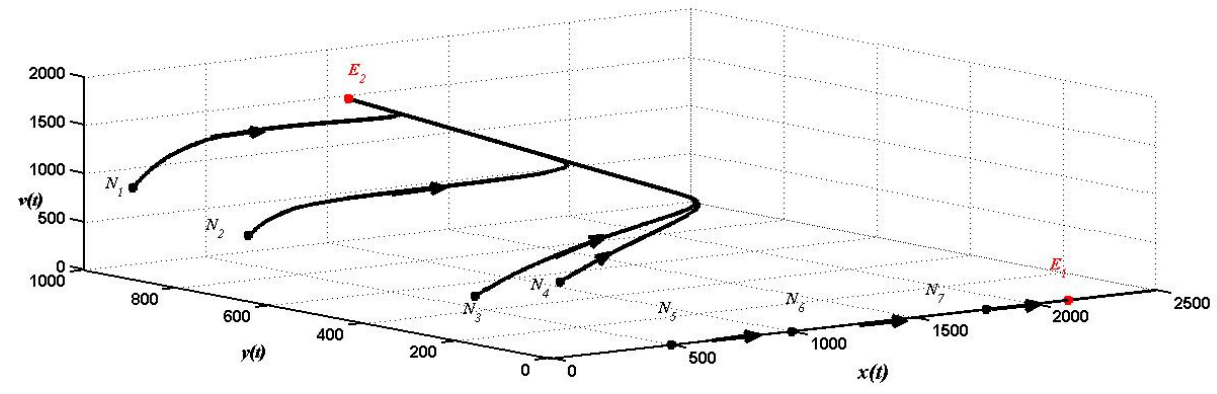

(a)

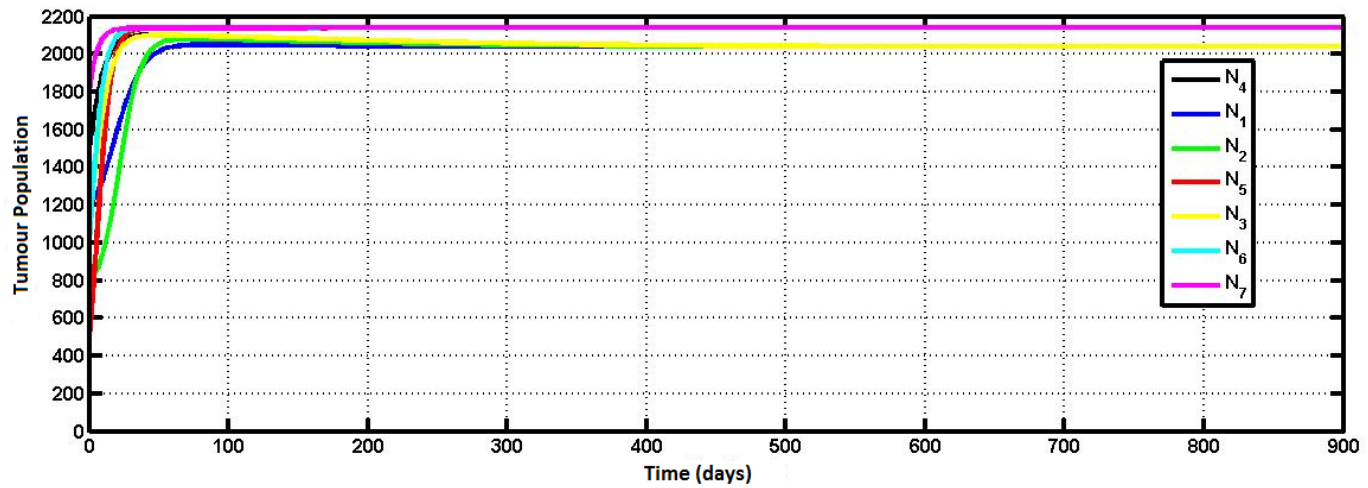

(b)

Figure 2. The phase portrait showing that E2 is local asymptotically stable (a). Number of total tumour cells every time, for $0 \leq \mathrm{t}$ $\leq 900($ b).

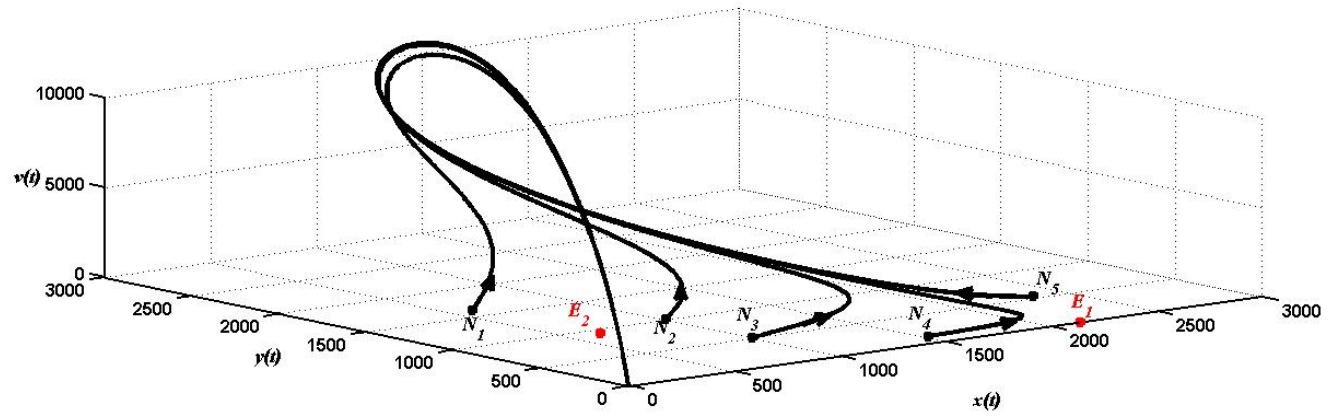

Figure 3. The portrait of phase showing equilibrium E1 and E2 unstable

Similar with the first simulation, the total tumour cell during the time execution also never exceeded the maximum number of tumour cells inside the human body, as presented in Figure $2 \mathrm{~b}$.

The third simulation is conducted to investigate what happen if both of equilibrium points, $E_{1}$ and $E_{2}$ are unstable. We adjust the parameter such that equilibrium point $E_{2}$ exist, but it is unstable. By taking $\mathrm{r}=$ $0.2, \mathrm{~K}=2139, \beta=0.0127, \delta=0.01, \gamma=0.0125$ and $\mathrm{b}=$ 20 we have $E_{1}=(2139,0,0)$ and $E_{2}=(9,171,261)$, but $A_{1} A_{2}-A_{3}>0$. Therefore the stability condition of $E_{1}$ and $E_{2}$ are not fulfilled. We deliver the result of numerical simulation in Figure 3, which shows some orbits of solution that start from the following initial values: $\mathrm{N}_{1}$ $=(500,1500,400), \mathrm{N}_{2}=(1000,1000,100), \mathrm{N}_{3}=(1000$, $500,50), \mathrm{N}_{4}=(1500,100,100), \mathrm{N}_{5}=(2000,100,1500)$. It can be seen that all orbits neither move toward $E_{1}$ nor $E_{2}$, but move toward $(0,0,0)$. Medically speaking, this interesting result indicates that we can adjust the parameter values to reach virotherapy goal, namely the total tumour cells, including uninfected as well as infected tumour cells is destroyed. 


\section{CONCLUSION}

In this work, a dynamical analysis has been conducted on a modified model of tumour virotherapy using the oncolytic virus, where the bilinear incident rate is replaced by standard incident rate. There are two equilibrium points of the model, namely uninfected equilibrium and endemic equilibrium. The uninfected equilibrium always exists while the endemic equilibrium exists under an existence condition, indicated by a positive dimensionless basic reproduction number. Moreover this number also determines the stability of the equilibrium points. When the basic reproduction number is less than one, the uninfected equilibrium point is global asymptotically stable. Otherwise, the endemic equilibrium exists, but it still needs a condition to be local asymptotically stable. The result of numerical simulations agrees with the dynamical analysis. Moreover, it can be shown by numerical simulation that there is a set of parameter values that can simulate a situation when the tumour cells become disappear.

\section{ACKNOWLEDGMENT}

\section{REFERENCES}

1. Tian JP (2011) The Replicability of oncolytic virus: Defining conditions in tumour virotherapy. Mathematical Biosciences and Engineering 8 (3): 841-860.
2. Wodarz D (2003) Gene therapy for killing p53-negative cancer cells: use of replicating versus nonreplicating agents. Human Gene Therapy 14 (2): 153-159.

3. Dingli D, Cascino MD, Josic K et al. (2006) Mathematical modeling of cancer radio virotherapy. Mathematical Biosciences 199 (1): 55-78.

4. Nagle RK, Saff EB, Snider AD (2012) Fundamentals of differential equation. New York, Pearson.

5. Dingli D, Carr T, Josic K, Russel JS (2008) Modeling of cancer virotherapy with recombinant measles viruses. Journal Theoretical Biology 252 (1): 109-122.

6. Min L, Su Y, Kuang Y (2008) Mathematical analysis of a basic model of virus infection with application to HBV infection. Rocky Mountain Journal of Mathematics 38 (5): 1573-1585.

7. Mattheij R, Molenaar J (2002) Ordinary differential equation in theory and practice. New York, Society for Industrial and Applied Mathematics.

8. Alligood KT, Sauer TD, Yorke JA (2000) CHAOS: An introduction to dynamical systems. New York, SpringerVerlag.

9. Panfilov A (2004) Qualitative Analysis of Differential equation. Utrecht, Utrecht University.

10. Nowak M, May RM (2000) Virus dynamics: Mathematical principles of immunology and virology. Oxford, Oxford University Press.

11. Murray JD (2002) Mathematical biology: An introduction, third edition. Berlin, Springer-Verlag, Inc. 